observed with temperature was attributable to the increased number of binding sites for ANS which were available at the higher temperature. Thus there was evidently a change in head-group configuration with temperature. A comparison of the enthalpy change for the head-group transition, which was calculated from the Van't Hoff equation with published values for the whole process (involving heads and tails), led to the conclusion that the transition is a cooperative process. The "cooperativity factor" $(\sigma)$ was of the same order as that found for the helix-coil transition of polyglutamic acid. This large degree of cooperativity may be thought of as the nucleation of a two-dimensional "crystallization" of the head groups on cooling.

Changing the $p \mathrm{H}$ or calcium concentration was shown to alter or initiate the phase change and it was also shown that in the presence of multivalent ions and also, interestingly, of acetylcholine, the system exhibited hysteresis. Hysteresis means that a given state of a system depends on its past history and thus the system is, in a sense, capable of information storage. Such a hysteresis is also capable of giving rise to oscillatory fluxes in membranes. Träuble also used the temperature-jump method to investigate the rate of the phase change and found that the relaxation time could be markedly reduced by the presence of cholesterol in the lipid phase. With calcium in the aqueous solution as well, the relaxation time was further reduced to the order of $1 \mathrm{~ms}$, which is getting down towards the duration of a nerve impulse. This effect of cholesterol implies a new role for it in biological membranes-that it enables the membrane lipids to undergo a rapid phase change.

These lipid phase changes may be important in the function of the membranes of temperature receptors. Whether such processes are of any relevance in the function of nerve cells remains at present no more than an interesting speculation.

\section{RNA PHAGES}

\section{Progeny Release}

from our Cell Biology Correspondent

WE know more about the biology of R17, $Q \beta$ and the other RNA phagesthe simplest known phages-than any other group of viruses. The three genes which comprise their genome have been mapped and their functions defined, and two of the proteins specified by these genes have been sequenced. Large segments of the RNA of these viruses have been sequenced and it cannot be long before the entire sequence of the $\mathrm{R} 17$ or $\mathrm{Q} \beta$ genome has been elucidated.
The replicase of phage $\mathrm{Q} \beta$ has been isolated pure and used to replicate the $\mathrm{Q} \beta$ genome in vitro. Phage particles have been assembled in vitro from coat protein, assembly protein and RNA, and the strategy of the viral replication, the way in which ribosomes, coat protein and replicase compete for the viral RNA has been worked out. With so much already under their belts, molecular biologists might be forgiven for regarding the RNA phages as a field of research with nothing much of interest left to be discovered. While it is true that what remains to be found is perhaps unlikely to be as exciting as that found already, the results of recent work by Engelberg and Soudry (J. Virology, 8,257 ; 1971) and Passent and Kaesberg (ibid., 286) justify their persistence with these phages.

Engelberg and Soudry have tackled the problem of the release of progeny $\mathrm{Q} \beta$ phages from infected cells; a pro. cess which does not involve a lysozymelike enzyme specified by the phage. At $37^{\circ} \mathrm{C}$ infected $E$. coli lyse, whereas at $30^{\circ} \mathrm{C}$ infected cells liberate progeny $\mathrm{Q} \beta$ particles without being lysed. This nonlytic release is reminiscent of the release of the filamentous DNA male specific phages, first discovered by Hoffman et al. working with MS2, and suggests that the host contributes to the release mechanism. Engelberg and Soudry have now obtained direct evidence that this is the case by using chloramphenicol and rifampin to inhibit host protein and RNA synthesis, respectively. Both

\section{Brain and Brawn}

ThE fine microfilaments which so abound in nerve cells and are particularly prominent near the tips of growing axons are made of actin or, to be more precise, of a protein with striking chemical similarity to muscle actin. That is the remarkable conclusion drawn by Fine and Bray from a series of elegant experiments reported in next Wednesday's Nature New Biology. They have isolated the two chief proteins from neurones taken from chick sympathetic ganglia and maintained in culture. These two proteins each account for about 20 per cent of the total protein and one, with a molecular weight of about 55,000 , is tubulin, the subunit protein of microtubules. The second major protein, which has a molecular weight of about 45,000 , co-purifies and co-electrophoreses on SDS acrylamide gels with muscle actin.

Fingerprints of this protein labelled with $35 \mathrm{~S}$ methionine can be almost completely superimposed on fingerprints of chick breast muscle actin. The two fingerprints are not completely identical, however, and so at this stage there remains the possibility that the neurone these drugs inhibit the release of progeny phage irrespective of the temperature at which the cells are cultured. Inhibition is not immediate but occurs within about 15 minutes at $37^{\circ} \mathrm{C}$ and 30 minutes at $30^{\circ} \mathrm{C}$, suggesting that a labile host protein is involved. Further evidence for this conclusion comes from the finding that rifampin does not inhibit the release of $\mathrm{Q} \beta$ from infected, mutant cells which are resistant to the drug because they contain a rifampin resistant DNA dependent RNA polymerase (DNA transcriptase). Such resistant mutants continue to make messenger RNA in the presence of rifampin, and also to release $\mathrm{Q} \beta$. Engelberg and Soudry reasonably conclude, therefore, that a protein specified by a host cell gene is required for continued release of this phage. Short lived protein appendages of male bacteria, the F pili, are the obvious candidates for the release protein.

Passent and Kaesberg have found that in the presence of rifampin, progeny $\mathrm{Q} \beta$ particles are not released because, they postulate, particles are not assembled. Their experiments show that in the presence of rifampin $\mathrm{Q} \beta$, RNA is replicated normally, coat protein is made in almost the same amounts as in the drug's absence (as is the assembly protein) but the yield of phage particles is decreased by about 95 per cent. When resistant $E$. coli are used, the yield is unaffected by the drug. Rather than invoke some failure in a release mechanism, Passent and

actin is not identical to muscle actin but the two proteins cannot differ by very much, if at all.

As Fine and Bray comment, it is not perhaps particularly surprising that nerve cells contain large amounts of actin, for axons grow at rates as high as $50 \mu \mathrm{m}$ per hour and the tip of the growing axon continually undulates and such motility presumably demands some form of contractile apparatus. They have also obtained evidence which indicates that actin or an actin like protein is in chick embryo lens, lung, skin, heart, pancreas and kidney cells. Furthermore, fingerprints of chick embryo brain actin are so similar to those of muscle actin that the two proteins may well be identical although, as Fine and Bray say, "nothing short of the total amino-acid sequences of the two proteins will establish complete identity".

It seems therefore that actin may be universal to all cells at some stage in their development, the intracellular location and precise function of the protein being in part determined by the other proteins with which it interacts. 